\title{
Role of DISC1 in Neuronal Trafficking and its Implication in Neuropsychiatric Manifestation and Neurotherapeutics
}

\author{
Toshifumi Tomoda $^{1}$ (D) Takatoshi Hikida ${ }^{2} \cdot$ Takeshi Sakurai $^{3}$
}

Published online: 29 June 2017

(C) The American Society for Experimental NeuroTherapeutics, Inc. 2017

\begin{abstract}
Disrupted-in-schizophrenia 1 (DISC1) was initially identified as a gene disrupted by a translocation mutation cosegregating with a variety of psychotic and mood disorders in a Scottish pedigree. In agreement with this original finding, mouse models that perturb Discl display deficits of behaviors in specific dimensions, such as cognition and emotion, but not a motor dimension. Although DISCl is not a risk gene for sporadic cases of specific psychiatric disorders defined by categorical diagnostic criteria (e.g., schizophrenia and major depressive disorder), DISC1 is now regarded as an important molecular lead to decipher molecular pathology for specific dimensions relevant to major mental illnesses. Emerging evidence points to the role of DISC1 in the regulation of intracellular trafficking of a wide range of neuronal cargoes. We will review recent progress in this aspect of DISC1 biology and discuss how we could utilize this body of knowledge to better understand the pathophysiology of mental illnesses.
\end{abstract}

Keywords DISC1 $\cdot$ Trafficking $\cdot$ Interactome $\cdot$ Cargo $\cdot$ Schizophrenia

Electronic supplementary material The online version of this article (doi:10.1007/s13311-017-0556-5) contains supplementary material, which is available to authorized users.

Toshifumi Tomoda ttomoda1@gmail.com

1 Centre for Addiction and Mental Health, University of Toronto, Toronto, Canada

2 Laboratory for Advanced Brain Functions, Institute for Protein Research, Osaka University, Osaka, Japan

3 Medical Innovation Center, Kyoto University Graduate School of Medicine, Kyoto, Japan

\section{Introduction}

Since the discovery of disrupted-in-schizophrenia 1 (DISC1) as a gene disrupted by a balanced chromosomal translocation $(1 ; 11)(q 42.1 ; \mathrm{q} 14.3)$ in a large Scottish kindred associated with hereditary mental illnesses, including both depression and schizophrenia, a number of cell and molecular studies have reported on the roles of DISC1 in neurodevelopment and synaptic functions [1]. These studies have laid the groundwork for biological hypothesis of major mental illnesses. Subsequently, mouse models mimicking $\mathrm{DISCl}$ dysfunction successfully demonstrated behavioral deficits in cognitive and emotional dimensions, satisfying face validity of these mice as models of a wide range of human psychiatric conditions [2]. Recent genome-wide association studies with large cohorts of patients with schizophrenia and bipolar disorder have failed to identify DISCl as a promising gene associated with sporadic cases of a specific condition [3], which is in accordance with the results of meta-analyses [4]. Although it has become evident that DISC1 no longer serves as a risk gene for specific psychiatric disorders, which are defined by categorical diagnostic criteria that are currently used (e.g., schizophrenia and major depressive disorder), DISC1 continues to be regarded as a molecular lead to decipher molecular pathology underlying specific dimensions relevant to major mental illnesses [5].

Accumulating evidence from cell biology studies has provided a new glimpse of this gene as an attractive lead useful not only for understanding the pathophysiological processes, but also for developing novel treatment options for the disease. Of note, a number of proteins that bind DISC1 have turned out to be components of intracellular trafficking machinery in neurons, as will be discussed in detail in the following sections. DISC1 appears to function as an adaptor that links a wide variety of neuronal cargoes to respective motor machinery, through interaction with its multiple binding 
partners (Fig. 1). This view proposes DISC1 as a central coordinator of neuronal trafficking that enables proper delivery of a range of cargoes with spatial and temporal precision, thereby ensuring normal neuronal development, as well as functional homeostasis. While the role of DISC1 in neurodevelopment has been extensively studied in the past literature [6], a series of recent reports also emphasize the role of DISC1 in the functional regulation of synapses at postdevelopmental stages [7-14]. However, mechanistic underpinnings of how DISC1 regulates both neurodevelopment and postdevelopmental synaptic functions remain to be understood.

Here we discuss the possibility that the DISC1-containing neuronal trafficking machinery provides a unified platform for regulating both neurodevelopment and neuronal functions, which likely helps us understand the disease trajectory based on such a unified molecular mechanism.

\section{DISC1 Interactome}

Because DISC1 has no significant sequence homology to any functional domains with particular enzymatic activities or a known functional motif, attempts were made to predict the functional property by identifying its binding partners through a series of yeast 2-hybrid screens and co-immunoprecipitation assays. As previously summarized [1], a plethora of proteins were identified in these initial efforts, including cytoskeletonor centrosome-associated proteins (e.g., nudE-like 1, lissencephaly-1, microtubule-associated protein $1 \mathrm{~A}, \beta 4-$

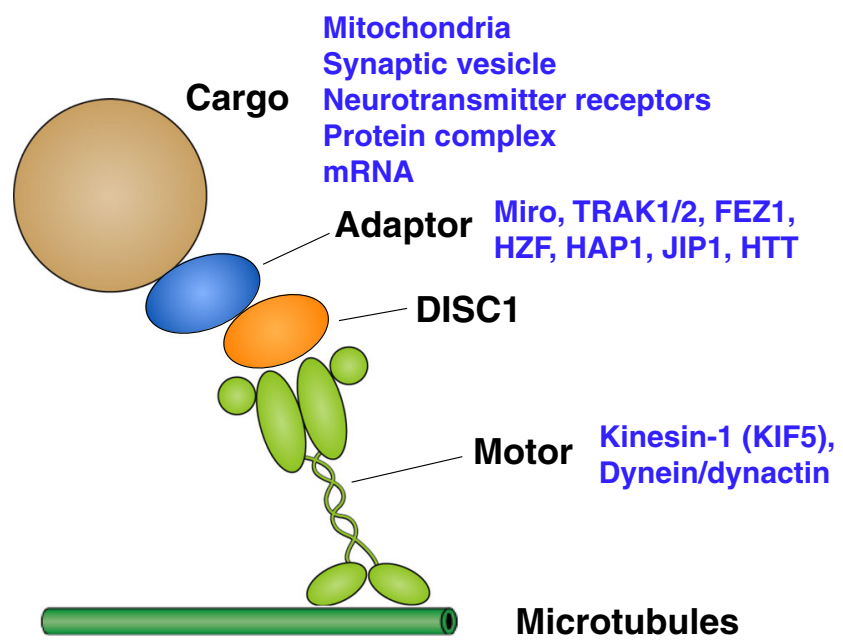

Fig. 1 Disrupted-in-schizophrenia 1 (DISC1)-containing neuronal trafficking machinery. Schematic view of the multiple components of trafficking machinery regulated by DISC1, which includes cargoes, adaptor molecules, motor proteins, and cytoskeletal components. TRAK $1 / 2$ = trafficking kinesin-binding protein $1 / 2 ; \mathrm{FEZ} 1$ = fasciculation and elongation protein zeta; HZF $=$ hematopoietic zinc finger; HAP1 = huntingtin-associated protein $1 ; \mathrm{JIP} 1=\mathrm{JNK}$-interacting protein 1 ; HTT $=$ Huntingtin spectrin, Bardet-Biedl Syndrome 4), motor proteins (e.g., kinesin-related protein 5 , dynein/dynactin), adaptor proteins [e.g., fasciculation and elongation protein zeta 1 (FEZ1), Huntingtin-associated protein 1], and synaptic enzymes (e.g., Kalirin-7). DISC1 appears to have a modular structure, in which each part of the protein serves as a binding domain for respective binding partners (as shown in Fig. 2). More extensive yeast 2 -hybrid screens coupled with pathway analyses revealed the DISC1 interactome [19], which further implicated DISC1 in intracellular transport processes along the cytoskeletal network. These studies collectively supported the idea that DISC1 controls neurodevelopment and synapse maturation via regulation of neuronal trafficking processes.

\section{DISC1 as a Neuronal Cargo Adaptor}

Subsequent studies continued to provide a wider view of the role of DISC1 in neuronal trafficking. In particular, types of cargoes reported to be transported by DISC1-containing trafficking machinery now range from organelles to mRNA to neurotransmitter receptors (Table 1, Fig. 1). In each case, DISC1 appears to serve as an adaptor that links a cargo to a specific motor complex by recruiting an additional set of adaptor proteins. It is also becoming evident that DISC1 regulates both short- (i.e., within soma) and long-distance (i.e., between soma and axons or dendrites) trafficking, depending on subcellular localization of each cargo or on local needs inside neurons. In the following section, we will summarize the components and the regulatory modes of DISC1-mediated trafficking of a series of cargoes.

\section{Mitochondria}

DISC1 regulates mitochondrial dynamics (trafficking, fission, and fusion), which is shown to be essential for synaptogenesis and neurotransmission [30]. Direct evidence that DISC1 plays a role in mitochondrial transport came from a study showing that overexpression or depletion of DISC1 caused an increase or a decrease, respectively, in the proportion of motile mitochondria in axonal and dendritic compartments of cultured neurons [31]. This finding is in good agreement with the notion that DISC1 functions in synaptic regulation, as a previous study implicated the role of mitochondrial transport in morphogenesis and plasticity of spines and synapses [32]. Molecular evidence showed that DISC1 interacts with both Miro, a mitochondrial resident protein with calcium-binding and guanosine triphosphatase-like domains critical to mitochondrial transport [33, 34], and trafficking kinesin-binding protein 1/2 (TRAK1/2) key trafficking adaptor proteins critical for linking cargoes to kinesin motors [35], thereby forming a protein complex responsible for efficient mitochondrial transport [20,21]. Miro interacts with the N-terminal domain 


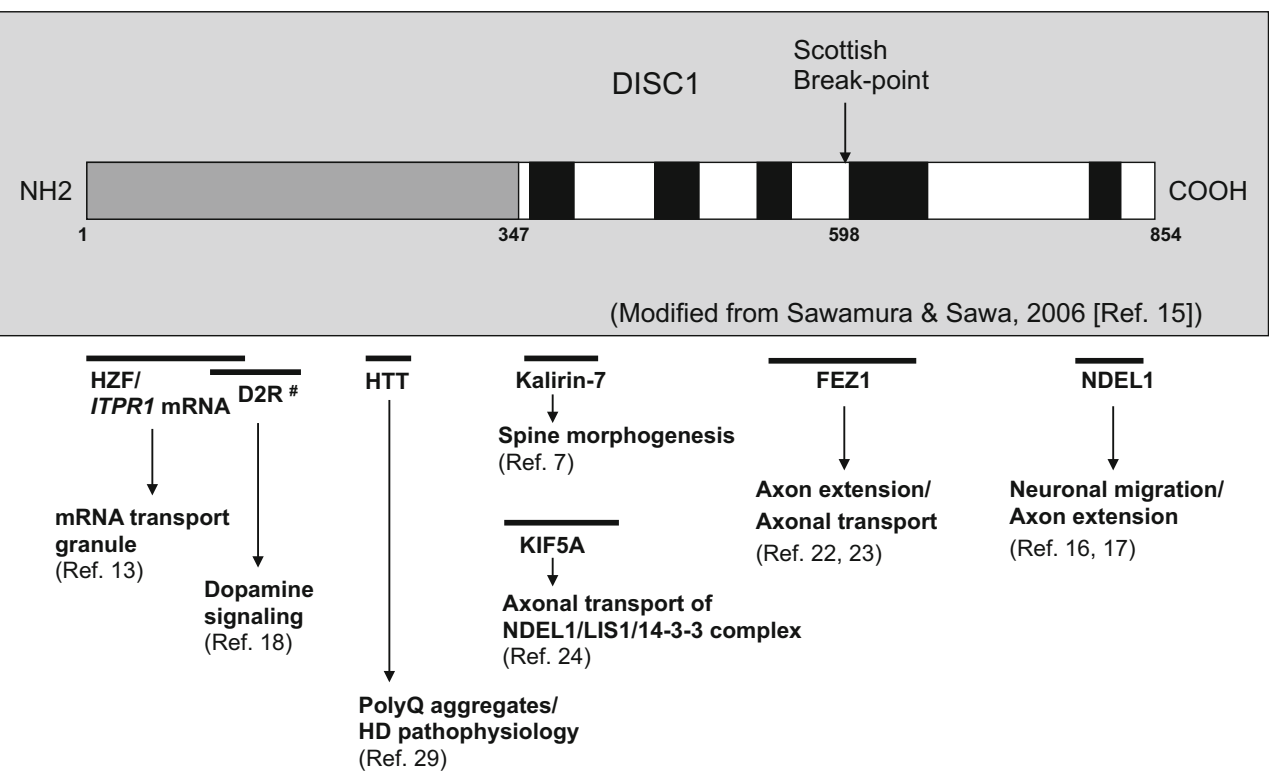

Fig. 2 Disrupted-in-schizophrenia 1 (DISC1)-interacting proteins that function in neuronal trafficking. Among a number of DISC1 binding partners, several representative molecules that function in neuronal trafficking or serve as cargoes are depicted. Horizontal bars indicate approximate region of DISC1 protein that interacts with its respective binding partners shown underneath each bar. HZF = hematopoietic zinc finger; HTT $=$ Huntingtin; PolyQ = polyglutamine; KIF5A = kinesin

of DISC1, and expressing a DISC1 point mutant within this domain (R37W) impairs anterograde transport of mitochondria in axons. Importantly, expressing the DISC1-Miro interacting domain or the $\mathrm{C}$-terminal deletion construct of DISC1, which corresponds to a putative truncated DISC1 mutant speculated to be produced by the Scottish translocation mutation, inhibited dendritic outgrowth in cultured neurons [21]. Consistently, a similar decrease in dendritic complexity has been reported in a series of DISC1 mutant mouse models [7, 36-39], as well as in postmortem brain samples from psychiatric patients [40]. Additionally, DISC1 is reported to heavy chain isoform 5A; NDEL1 = nudE-like 1; LIS1 = lissencephaly$1 ; \mathrm{FEZ1}=$ fasciculation and elongation protein zeta. ${ }^{\text {\#The exact domain }}$ of DISC1 that interacts with dopamine D2 receptor (D2R) has not been mapped in the original literature [18], but Disc1 L100P mutation has been shown to influence Disc1-D2R interaction, suggesting that the Nterminal domain encompassing this point mutation is presumed to provide at least a part of binding interface with D2R

collaborate with syntaphilin, an axonally targeted anchor protein, to control mitochondrial transport for axonal branching and function [41]. Thus, these lines of evidence suggest that deficits in DISC1-mediated mitochondrial transport impair neurodevelopment and contribute to susceptibility to psychiatric manifestation. It remains to be studied whether this mechanism for DISC1-mediated mitochondrial transport also plays a direct role in controlling synaptic functions in mature synapses at postdevelopmental stages. Because mitochondria are critical sensors and regulators of intracellular calcium signaling, and thus critical for synaptic functions [42], and

Table 1 Components of neuronal trafficking machinery regulated by Disrupted-in-schizophrenia 1 (DISC1)

\begin{tabular}{lllll}
\hline Cargo & Motor & Adaptor (additional regulators) & Subcellular compartment & Reference(s) \\
\hline Mitochondria & KIF5, dynein/dynactin & Miro, TRAK1/2 & Soma, axon, synapse & [20, 21] \\
$\begin{array}{l}\text { Synaptic vesicle (synaptotagmin-1) } \\
\text { mRNA granule }\end{array}$ & KIF5 & FEZ1 & Axon & Soma, dendrite \\
Protein complex (NDEL1/LIS1/14-3-3e) & KIF5 & HZF & Soma, axon & {$[13]$} \\
NMDA receptor & & PSD-95 (Kalirin-7, Rac1) & Excitatory postsynapse & {$[7,10]$} \\
GABA receptor & KIF5 & HAP1 & Inhibitory postsynapse & {$[25-27]$} \\
DR2 & & & Dopaminergic postsynapse & {$[18]$} \\
APP & KIF5 & JIP1 & Soma, axon & S28] \\
PDE4 & KIF5? & HTT & Soma
\end{tabular}

KIF5 = kinesin-related protein 5; TRAK1/2 = trafficking kinesin-binding protein 1/2; FEZ1 = fasciculation and elongation protein zeta; HZF $=$ hematopoietic zinc finger; NDEL1 = nudE-like 1 ; LIS1 $=$ lissencephaly-1; NMDA $=N$-methyl-D-aspartate; PSD-95 = postsynaptic density protein 95 ; GABA $=\gamma$-aminobutyric acid; HAP1 $=$ Huntingtin-associated protein 1 ; DR2 $=$ dopamine D2 receptor; APP $=$ amyloid precursor protein; JIP1 $=$ JNK-interacting protein 1 ; PDE4 = phosphodiesterase 4; HTT = Huntingtin 
aberrant calcium homeostasis due to mitochondrial dysfunction has been reported for neuropsychiatric diseases, including autism [43], it is worth studying the role of DISC1 in mitochondrial calcium dynamics. In this regard, it is of note that DISC1 regulates endoplasmic reticulum calcium dynamics via interaction with EXOC, a subunit of the exocyst complex, and inositol-1,4,5-trisphosphate receptor 1 (ITPR1) which plays a critical role in synaptic plasticity [44].

\section{Synaptic Vesicle}

DISC1 also plays a role in transport of synaptic vesicle (SV) precursors in cultured neurons [22]. DISC1 knockdown or expression of the truncated Scottish DISC1 mutant in cultured neurons caused a decrease in the proportion of motile SV precursors. Molecular evidence showed that DISC1 interacts with FEZ1 adaptor protein [23], and stabilizes the complex formation between FEZ1 and synaptotagmin-1, a SV-resident protein important for the regulation of neurotransmitter release [22]. Consistently, defects in axonal morphology and presynaptic firing rate have been observed in Discl mutant mice that carry a putative C-terminal truncation mutation in Discl locus mimicking the Scottish translocation mutation [37]. Furthermore, deficits in SV release were also reported in human induced pluripotent stem cell-derived forebrain neurons that harbor a frame shift mutation in DISC1 [11]. Collectively, the results suggest a possible role of DISC1-mediated SV transport in the regulation of axonal and presynaptic functions.

\section{Amyloid Precursor Protein}

A recent report showed that DISC1 regulates trafficking and processing of amyloid precursor protein (APP), a neuronal vesicle-resident transmembrane protein implicated in the pathophysiology of Alzheimer's disease [28]. DISC1 knockdown in cultured neurons caused a decrease in APP internalization and a reciprocal increase in surface expression of APP, leading to alteration in proteolytic APP processing and amyloid beta generation. Molecular studies established that APP transport in axons is regulated by kinesin-1 motor and its adaptor c-Junamino-terminal kinase-interacting protein 1 [45]. It remains to be tested whether the known machinery for APP trafficking could work in concert with DISC1-mediated mechanisms to regulate APP trafficking and processing. Nonetheless, the mechanisms underlying DISC1-APP interaction may provide an opportunity to test whether APP serves as a modifier of neuropsychiatric conditions regulated by DISC1 function. Alternatively, this could also provide a molecular clue that may contribute to deeper understanding of deficits in neurocognitive processes in neurodegenerative diseases.

\section{mRNA}

A recent nonbiased proteomic approach identified a several RNA-binding proteins, including hematopoietic zinc finger (HZF), as an additional class of DISC1 binding partners [13]. HZF interacts with several mRNAs, including the one for ITPR1. DISC1 enhances the association of ITPRI mRNA/ HZF-containing granule with kinesin-1 to deliver the complex toward the dendrite. Loss of function of DISC1 reduces the rate of dendritic transport of ITRP1 mRNA, resulting in impairment of synaptic plasticity.

\section{Components at the Excitatory and Inhibitory Postsynapses}

Emerging evidence highlights additional strategy for neurons to regulate neurotransmission via DISC1-dependent local trafficking mechanisms in both excitatory and inhibitory postsynapses. At the resting state of excitatory postsynapses, DISC1 forms a complex with postsynaptic density protein 95 and sequesters guanine exchange factor Kalirin-7, limiting access of Kalirin-7 to Rac1 guanosine triphosphatase. Upon $N$-methyl-D-aspartate receptor activation, Kalirin-7 is released from this complex and facilitates Rac1-guanosine triphosphate binding, resulting in p21 activated kinase phosphorylation and increased synaptic transmission [7].

Recent reports also suggest DISC1-dependent mechanisms for regulating the trafficking of $\gamma$-aminobutyric acid $(\mathrm{GABA})_{\mathrm{A}}$ receptors, which mediate fast inhibitory neurotransmission [46]. Disturbance in GABA system has been implicated in the pathophysiology of neuropsychiatric disorders, including schizophrenia. DISC1 knockdown caused a decrease in surface expression of $\mathrm{GABA}_{\mathrm{A}}$ receptors in cultured neurons, and concomitant reduction in $\mathrm{GABA}_{\mathrm{A}}$ receptormediated inhibitory currents $[14,25]$, as well as deficits in $\mathrm{GABA}_{\mathrm{A}}$ receptor-mediated dendritic development [25]. Because molecular evidence shows that trafficking of $\mathrm{GABA}_{\mathrm{A}}$ receptors are regulated in a kinesin-1-dependent manner [26, 27], it remains to be tested whether DISC1dependent mechanisms for $\mathrm{GABA}_{\mathrm{A}}$ receptor trafficking are linked to this motor machinery. Collectively, these studies suggest that DISC1 coordinates the excitatory-inhibitory balance at the circuit level via regulation of trafficking of both excitatory and inhibitory neurotransmitter receptors. This further raises a possibility that excitatory-inhibitory imbalance due to deregulated DISC1 function may underlie deficits in long-term potentiation or cortical plasticity, as observed in several Disc1 mutant mouse models [13, 39].

\section{Dopamine D2 Receptor}

In addition, DISC1 has been shown to couple with the dopamine D2 receptor (D2R), which serves as a main target of a 
majority of antipsychotic drugs currently available [18]. In this report, interaction of DISC1 with D2R was found to be enhanced in postmortem brain samples from sporadic cases of patients with schizophrenia, as well as in Disc1 L100P point mutant mice that exhibit schizophrenia-related endophenotypes, such as amphetamine-induced hyperlocomotion and disrupted prepulse inhibition. Molecular analysis demonstrated that greater levels of DISC1-D2R association stabilize D2R signaling by enhancing surface presentation of D2R. Conversely, inhibiting DISC1-D2R association in Disc1 L100P mice using specific interfering peptides downregulated the surface expression of D2R and ameliorated hyperlocomotion and disrupted prepulse inhibition. This work supported a notion that DISC1-D2R interaction has a causal role in psychiatric manifestation, and presented a potential avenue for a novel therapeutic approach against such conditions.

\section{Discussion and Future Therapeutic Perspective}

As described above, it is increasingly evident that DISC1 plays a broad role in regulating the trafficking of a wide range of cargoes at multiple neuronal compartments over the entire neuronal life span. It goes beyond our imagination how 1 protein could coordinate such multiple tasks in a temporally and spatially controlled manner. One strategy to determine cargo specificity is to use a distinct combination of adaptor proteins; we have described that DISC1 couples with Miro on the mitochondria, whereas FEZ1 couples with synaptotagmin1 on the SV. Another strategy that neurons could use is to modulate DISC1 functions by post-translational modifications, most notably via phosphorylation of a specific amino acid. For example, unphosphorylated DISC1 binds glycogen synthase kinase 3 beta and promotes neuronal proliferation via Wnt signaling regulation, whereas phosphorylated DISC1 (at serine-710) preferentially interacts with Bardet-Biedl syndrome proteins and recruits them to the centrosome to drive the migration of postmitotic neurons [47]. Additionally, phosphorylation of DISC1 at serine-58 enhances its affinity to activating transcription factor 4 [48], suggesting that phosphorylation may provide neurons with a strategy to alter DISC1 binding specificity. Another key issue to control DISC1-mediated trafficking is its propensity to form multimers or aggregates; modest overexpression of DISC1 in the nervous system is reported to trigger DISC1 multimerization and subsequent changes in the activity of D2R signaling [49]. Likewise, DISC1 point mutation S704C is shown to be prone to aggresome formation [50,51], which may negatively affect neuronal trafficking [52]. DISC1 is also reported to interact with Huntingtin (HTT), a protein that is aberrantly regulated in Huntington's disease (HD) and that also functions as a component of kinesin-1-dependent intracellular trafficking machinery $[29,53]$. In a recent study, mutant HTT proteins with polyglutamine tracts present in HD brains were shown to form aggregates with DISC1 and titrate out the functional pool of DISC1, thereby causing neuropsychiatric symptoms observed in HD [29]. This study underscores the critical roles of HTT and DISC1 in the regulation of neuronal trafficking, which may underlie part of HD pathophysiology.

Neuronal trafficking provides neurons with a means to ensure their viability and achieve highest levels of functional homeostasis. Although we now have a body of knowledge demonstrating that DISC1 regulates neuronal trafficking during neurodevelopment, as well as at functionally matured states, a question remains as to how DISC1 dysfunction may lead to psychiatric manifestation through deregulated neuronal trafficking. One possibility is that impaired DISC1 function during development would result in formation of malfunctioning neuronal circuit that is prone to psychiatric manifestation at later stages. An alternative view is that impaired DISC1 function during development may impose minimal influence on the development of the circuitry, and deterioration of the trafficking machinery at later stages, coupled with environmental stressors, would mainly drive psychiatric manifestation. In either case, dysfunction in DISC1-mediated trafficking mechanisms likely leads to circuit-level disturbance through deregulated trafficking of both excitatory and inhibitory neurotransmitter receptors. Besides, the roles of DISC1 in neuronal trafficking are consistent with and complementary to a previously proposed view that DISC1 functions as a signaling scaffold protein or a synapse integrator [1]. Both the signaling and synaptic components need to be transported to the appropriate subcellular compartments for their optimal functioning; deregulated trafficking of these components likely deteriorates neuronal functions, leading to psychiatric manifestation. Questions remain as to how this genetic deficit manifests as neuropsychiatric symptoms through homeostatic compensation of the affected brain network, as discussed for autism spectrum disorders [54].

Another question is whether and how we could manipulate this DISC1-mediated trafficking system to conceive potential therapeutic avenues against psychiatric disorders. Given a number of binding partners and pleiotropic effects of DISC1, targeting the trafficking machinery regulated by DISC1 is expected to have profound effects on neuronal functioning or viability, excluding such strategies as viable options. One possibility may be to achieve specificity of targeting by focusing on a specific interface between DISC1 and its binding partner, as exemplified by $\mathrm{Su}$ et al. [18]; targeting the function of only 1 binding partner could presumably minimize potential side effects of the intervention. Nonetheless, careful attention must be paid to any therapeutic interventions that might have potential influences on fundamental neuronal mechanisms, such as intracellular trafficking regulation. In the case of targeting DISC1 as a therapeutic 
lead, available DISC1 mouse models will prove useful in evaluating the efficacy, as well as predicting adversities, of such treatment options.

Mental illnesses, including schizophrenia and mood disorders, affects up to $5 \%$ of the population worldwide in a given year, with a lifetime prevalence of $\sim 20 \%$, presenting a major burden on personal lives, healthcare systems, and society at large [55]. Although prior studies show that these disorders occur through a complex interaction of multiple genetic and environmental risk factors, the underlying pathophysiological mechanisms remain only fragmentally understood, and treatment options with available neuroleptics or antidepressants are only effective in alleviating some of the symptoms in a subset of patients. Part of the reasons for this problem could be that current treatments are identified serendipitously as agents that improve a portion of abnormal behavioral symptoms without targeting underlying pathophysiology. It could also be because the causes of the disorders are multifactorialmixed etiologies of both genetic and environmental factors, leading to varying degrees of multiple symptomatologies. Under this circumstance, it might be difficult to treat mental illnesses with a single therapeutic regimen. It is therefore increasingly important to identify or develop new therapeutic avenues that current treatment options fail to target. In this regard, further characterization of detailed molecular mechanisms underlying DISC1-mediated neuronal trafficking would be the first step toward the goal.

Acknowledgements We thank Dr. Akira Sawa (Johns Hopkins University) for advice. This work was supported by grants from DOD/ CDMRP (W81XWH-11-1-0269) and CTF-DDI (TT), Japan Society for the Promotion of Science Grants-in-Aid for Scientific Research (15H04275, 16H06568 and $16 \mathrm{~K} 14579$ to $\mathrm{TH} ; 15 \mathrm{H} 01285$ and 16 K01948 to TS), Takeda Science Foundation (TH), the Naito Foundation (TH), and the Kato Memorial Trust for Nambyo Research (TH). This work was performed, in part, under the International Cooperative Research Program of Institute for Protein Research, Osaka University, ICRa-17-13. The authors declare no conflict of interest.

Required Author Forms Disclosure forms provided by the authors are available with the online version of this article.

\section{References}

1. Brandon NJ, Sawa A. Linking neurodevelopmental and synaptic theories of mental illness through DISC1. Nat Rev Neurosci 2011;12:707-722.

2. Tomoda T, Sumitomo A, Jaaro-Peled H, Sawa A. Utility and validity of DISC1 mouse models in biological psychiatry. Neuroscience 2016;321:99-107.

3. Farrell MS, Werge T, Sklar P, et al. Evaluating historical candidate genes for schizophrenia. Mol Psychiatry 2015;20:555-562.

4. Mathieson I, Munafo MR, Flint J. Meta-analysis indicates that common variants at the DISC1 locus are not associated with schizophrenia. Mol Psychiatry 2012;17:634-641.
5. Niwa M, Cash-Padgett T, Kubo KI, et al. DISC1 a key molecular lead in psychiatry and neurodevelopment: No-More Disrupted-inSchizophrenia 1. Mol Psychiatry 2016;21:1488-1489.

6. Narayan S, Nakajima K, Sawa A. DISC1: a key lead in studying cortical development and associated brain disorders. Neuroscientist 2013;19:451-464.

7. Hayashi-Takagi A, Takaki M, Graziane N, et al. Disrupted-inSchizophrenia 1 (DISC1) regulates spines of the glutamate synapse via Rac1. Nat Neurosci 2010;13:327-332.

8. Wang Q, Charych EI, Pulito VL, et al. The psychiatric disease risk factors DISC1 and TNIK interact to regulate synapse composition and function. Mol Psychiatry 2011;16:1006-1023.

9. Hayashi-Takagi A, Araki Y, Nakamura M, et al. PAKs inhibitors ameliorate schizophrenia-associated dendritic spine deterioration in vitro and in vivo during late adolescence. Proc Natl Acad Sci U S A 2014;111:6461-6466.

10. Wei J, Graziane NM, Wang H, et al. Regulation of N-methyl-Daspartate receptors by disrupted-in-schizophrenia-1. Biol Psychiatry 2014;75:414-424.

11. Wen Z, Nguyen HN, Guo Z, et al. Synaptic dysregulation in a human iPS cell model of mental disorders. Nature 2014;515:414418.

12. Seshadri S, Faust T, Ishizuka K, et al. Interneuronal DISC1 regulates NRG1-ErbB4 signalling and excitatory-inhibitory synapse formation in the mature cortex. Nat Commun 2015;6:10118.

13. Tsuboi D, Kuroda K, Tanaka M, et al. Disrupted-in-schizophrenia 1 regulates transport of ITPR1 mRNA for synaptic plasticity. Nat Neurosci 2015;18:698-707.

14. Wei J, Graziane NM, Gu Z, Yan Z. DISC1 Protein regulates gamma-aminobutyric acid, type $\mathrm{A}\left(\mathrm{GABA}_{\mathrm{A}}\right)$ receptor trafficking and inhibitory synaptic transmission in cortical neurons. J Biol Chem 2015;290:27680-27687.

15. Sawamura N, Sawa A. Disrupted-in-schizophrenia-1 (DISC1): a key susceptibility factor for major mental illnesses. Ann N Y Acad Sci 2006;1086:126-133.

16. Ozeki Y, Tomoda T, Kleiderlein J, et al. Disrupted-inSchizophrenia-1 (DISC-1): mutant truncation prevents binding to NudE-like (NUDEL) and inhibits neurite outgrowth. Proc Natl Acad Sci U S A 2003;100:289-294.

17. Kamiya A, Kubo K, Tomoda T, et al. A schizophrenia-associated mutation of DISC1 perturbs cerebral cortex development. Nat Cell Biol 2005;7:1167-1178.

18. Su P, Li S, Chen S, et al. A dopamine D2 receptor-DISC1 protein complex may contribute to antipsychotic-like effects. Neuron 2014;84:1302-1316.

19. Camargo LM, Collura V, Rain JC, et al. Disrupted in Schizophrenia 1 Interactome: evidence for the close connectivity of risk genes and a potential synaptic basis for schizophrenia. Mol Psychiatry 2007;12:74-86.

20. Ogawa F, Malavasi EL, Crummie DK, et al. DISC1 complexes with TRAK1 and Miro1 to modulate anterograde axonal mitochondrial trafficking. Hum Mol Genet 2014;23:906-919.

21. Norkett R, Modi S, Birsa N, et al. DISC1-dependent regulation of mitochondrial dynamics controls the morphogenesis of complex neuronal dendrites. J Biol Chem 2016;291:613-629.

22. Flores R, 3rd, Hirota Y, Armstrong B, Sawa A, Tomoda T. DISC1 regulates synaptic vesicle transport via a lithium-sensitive pathway. Neurosci Res 2011;71:71-77.

23. Miyoshi K, Honda A, Baba K, et al. Disrupted-In-Schizophrenia 1, a candidate gene for schizophrenia, participates in neurite outgrowth. Mol Psychiatry 2003;8:685-694.

24. Taya S, Shinoda T, Tsuboi D, et al. DISC1 regulates the transport of the NUDEL/LIS1/14-3-3epsilon complex through kinesin-1. J Neurosci 2007;27:15-26.

25. Saito A, Taniguchi Y, Rannals MD, et al. Early postnatal GABAA receptor modulation reverses deficits in neuronal maturation in a 
conditional neurodevelopmental mouse model of DISC1. Mol Psychiatry 2016;21:1449-1459.

26. Twelvetrees AE, Yuen EY, Arancibia-Carcamo IL, et al. Delivery of GABAARs to synapses is mediated by HAP1-KIF5 and disrupted by mutant huntingtin. Neuron 2010;65:53-65.

27. Nakajima K, Yin X, Takei Y, Seog DH, Homma N, Hirokawa N. Molecular motor KIF5A is essential for GABA(A) receptor transport, and KIF5A deletion causes epilepsy. Neuron 2012;76:945961.

28. Shahani N, Seshadri S, Jaaro-Peled H, et al. DISC1 regulates trafficking and processing of APP and Abeta generation. Mol Psychiatry 2015;20:874-879.

29. Tanaka M, Ishizuka K, Nekooki-Machida Y, et al. Aggregation of scaffolding protein DISC1 dysregulates phosphodiesterase 4 in Huntington's disease. J Clin Invest 2017;127:1438-1450.

30. Murphy LC, Millar JK. Regulation of mitochondrial dynamics by DISC1, a putative risk factor for major mental illness. Schizophr Res 2017.

31. Atkin TA, MacAskill AF, Brandon NJ, Kittler JT. Disrupted in Schizophrenia-1 regulates intracellular trafficking of mitochondria in neurons. Mol Psychiatry 2011;16:122-124.

32. Li Z, Okamoto K, Hayashi Y, Sheng M. The importance of dendritic mitochondria in the morphogenesis and plasticity of spines and synapses. Cell 2004;119:873-887.

33. Wang X, Schwarz TL. The mechanism of $\mathrm{Ca}^{2}+$-dependent regulation of kinesin-mediated mitochondrial motility. Cell 2009;136: 163-174.

34. Macaskill AF, Rinholm JE, Twelvetrees AE, et al. Miro1 is a calcium sensor for glutamate receptor-dependent localization of mitochondria at synapses. Neuron 2009;61:541-555.

35. Brickley K, Stephenson FA. Trafficking kinesin protein (TRAK)mediated transport of mitochondria in axons of hippocampal neurons. J Biol Chem 2011;286:18079-18092.

36. Shen S, Lang B, Nakamoto C, et al. Schizophrenia-related neural and behavioral phenotypes in transgenic mice expressing truncated Disc1. J Neurosci 2008;28:10893-10904.

37. Kvajo M, McKellar H, Drew LJ, et al. Altered axonal targeting and short-term plasticity in the hippocampus of Disc1 mutant mice. Proc Natl Acad Sci U S A 2011;108:E1349-E1358.

38. Lepagnol-Bestel AM, Kvajo M, Karayiorgou M, Simonneau M, Gogos JA. A Disc1 mutation differentially affects neurites and spines in hippocampal and cortical neurons. Mol Cell Neurosci 2013;54:84-92.

39. Greenhill SD, Juczewski K, de Haan AM, Seaton G, Fox K, Hardingham NR. Adult cortical plasticity depends on an early postnatal critical period. Science 2015;349:424-427.

40. Kulkarni VA, Firestein BL. The dendritic tree and brain disorders. Mol Cell Neurosci 2012;50:10-20.
41. Park C, Lee SA, Hong JH, et al. Disrupted-in-schizophrenia 1 (DISC1) and Syntaphilin collaborate to modulate axonal mitochondrial anchoring. Mol Brain 2016;9:69.

42. Rizzuto R, De Stefani D, Raffaello A, Mammucari C. Mitochondria as sensors and regulators of calcium signalling. Nat Rev Mol Cell Biol 2012;13:566-578.

43. Sakurai T, Ramoz N, Barreto M, et al. Slc25a12 disruption alters myelination and neurofilaments: a model for a hypomyelination syndrome and childhood neurodevelopmental disorders. Biol Psychiatry 2010;67:887-894.

44. Park SJ, Jeong J, Park YU, et al. Disrupted-in-schizophrenia-1 (DISC1) regulates endoplasmic reticulum calcium dynamics. Sci Rep 2015;5:8694.

45. van der Kant R, Goldstein LS. Cellular functions of the amyloid precursor protein from development to dementia. Dev Cell 2015;32:502-515.

46. Smith KR, Kittler JT. The cell biology of synaptic inhibition in health and disease. Curr Opin Neurobiol 2010;20:550-556.

47. Ishizuka K, Kamiya A, Oh EC, et al. DISC1-dependent switch from progenitor proliferation to migration in the developing cortex. Nature 2011;473:92-96.

48. Soda T, Frank C, Ishizuka K, et al. DISC1-ATF4 transcriptional repression complex: dual regulation of the cAMP-PDE4 cascade by DISC1. Mol Psychiatry 2013;18:898-908.

49. Trossbach SV, Bader V, Hecher L, et al. Misassembly of full-length Disrupted-in-Schizophrenia 1 protein is linked to altered dopamine homeostasis and behavioral deficits. Mol Psychiatry 2016;21:15611572.

50. Leliveld SR, Bader V, Hendriks P, et al. Insolubility of disrupted-inschizophrenia 1 disrupts oligomer-dependent interactions with nuclear distribution element 1 and is associated with sporadic mental disease. J Neurosci 2008;28:3839-3845.

51. Narayanan S, Arthanari H, Wolfe MS, Wagner G. Molecular characterization of disrupted in schizophrenia-1 risk variant S704C reveals the formation of altered oligomeric assembly. J Biol Chem 2011;286:44266-44276.

52. Atkin TA, Brandon NJ, Kittler JT. Disrupted in Schizophrenia 1 forms pathological aggresomes that disrupt its function in intracellular transport. Hum Mol Genet 2012;21:2017-2028.

53. Boxall R, Porteous DJ, Thomson PA. DISC1 and Huntington's disease - overlapping pathways of vulnerability to neurological disorder? PLOS ONE 2011;6:e16263.

54. Nelson SB, Valakh V. Excitatory/inhibitory balance and circuit homeostasis in autism spectrum disorders. Neuron 2015;87:684-698.

55. World Health Organization. Shanghai declaration on promoting health in the 2030 Agenda for Sustainable Development. Health Promot Int 2017;32:7-8. 